\title{
Article \\ Gas Barrier Performance of Hexagonal Boron Nitride Monolayers Grown on Copper Foils with Electrochemical Polishing
}

\author{
Chil Hyoung Lee ${ }^{1,+}$, Go Bong Choi ${ }^{2,+} \mathbb{D}$, Eun Mi Kim ${ }^{1,3}$, Jongho Lee ${ }^{1} \mathbb{D}$, Jaegeun Lee ${ }^{4} \mathbb{D}$, Hi Gyu Moon ${ }^{5} \mathbb{D}$, \\ Myung Jong Kim ${ }^{6}\left(\mathbb{D}\right.$, Yoong Ahm Kim ${ }^{2, *(D)}$ and Tae Hoon Seo ${ }^{1, *(D)}$
}

1 Smart Energy \& Nano Convergence Research Group, Korea Institute of Industrial Technology, Gwangju 61012, Korea; chlee0901@kitech.re.kr (C.H.L.); kimeunmi@kitech.re.kr (E.M.K.); jholee@kitech.re.kr (J.H.)

2 Department of Polymer Engineering, Graduate School, Alan G. MacDiarmid Energy Research Institute \& School of Polymer Science and Engineering, Chonnam National University, Gwangju 61186, Korea; uppermost.peak@gmail.com

3 School of Materials Science \& Engineering, Chonnam National University, 77 Yongbong-ro, Buk-gu, Gwangju 61186, Korea

4 Department of Organic Material Science and Engineering, Pusan National University, 2, Busandaehak-ro 63 beon-gil, Geumjeong-gu, Busan 46241, Korea; jglee@pusan.ac.kr

5 Department of Inhalation Toxicology Research Center, Korea Institute of Toxicology, Jeongeup 56212, Korea; higyu.moon@kitox.re.kr

6 Department of Chemistry, Gachon University, 1342 Seongnam-daero, Sujeong-gu, Seongnam-si 13120, Korea; myungjongkim@gachon.ac.kr

check for updates

Citation: Lee, C.H.; Choi, G.B.; Kim, E.M.; Lee, J.; Lee, J.; Moon, H.G.; Kim, M.J.; Kim, Y.A.; Seo, T.H. Gas Barrier Performance of Hexagonal Boron Nitride Monolayers Grown on Copper Foils with Electrochemical Polishing. Appl. Sci. 2021, 11, 4599. https://doi.org/10.3390/ app11104599

Academic Editor: Simone Morais

Received: 15 April 2021

Accepted: 12 May 2021

Published: 18 May 2021

Publisher's Note: MDPI stays neutral with regard to jurisdictional claims in published maps and institutional affiliations.

Copyright: (c) 2021 by the authors. Licensee MDPI, Basel, Switzerland. This article is an open access article distributed under the terms and conditions of the Creative Commons Attribution (CC BY) license (https:/ / creativecommons.org/licenses/by/ $4.0 /)$.
* Correspondence: yak@chonnam.ac.kr (Y.A.K.); thseo@kitech.re.kr (T.H.S.)

+ These authors contributed equally to this work.

\begin{abstract}
The demand for high-performance two-dimensional gas barrier materials is increasing owing to their potential for application in optoelectronic devices. These materials can help the devices maintain their properties over a long period. Therefore, in this study, we investigated the gas barrier performance of hexagonal boron nitride (h-BN) monolayers grown on copper foils via electrochemical polishing (ECP). The ECP treatment helped reduce the surface roughness of the copper foils. As a result, the nucleation density was reduced and highly crystalline h-BN monolayers were produced. The gas barrier performance of h-BN monolayers on copper foils with ECP was comparable to that of graphene. Our finding demonstrates the potential of monolayer h-BN as a high-performance and economical gas barrier material for organic-based optoelectronic devices.
\end{abstract}

Keywords: two-dimensional; h-BN; Gas barrier; Electro-chemical polishing

\section{Introduction}

The development of organic-based optoelectronic devices has revolutionized the electronics industry owing to their superior material properties such as high quantum efficiency, high carrier mobility, good transparency, and high flexibility [1,2]. Despite the advantages of organic materials in optoelectronic device applications, their material properties become seriously degraded over time when they are exposed to water or oxygen [3]. Hence, the use of gas barriers is required to sustain their material properties. Though several materials have been suggested as candidates for gas barriers, such as metal, glass, $\mathrm{SiO}_{\mathrm{x}}$, and $\mathrm{Al}_{2} \mathrm{O}_{3}[4,5]$, two-dimensional (2D) materials have come into the limelight because they can utilize the properties of organic materials such as flexibility, lightness, and high optical transparency. Among various 2D materials, graphene has evident potential and is widely used as a gas barrier on account of its great barrier properties and its thermal and chemical stability [6,7].

However, graphene has limitations as a gas barrier. The most critical one is that it can facilitate the oxidation of materials [8]. According to recent reports, the high conductivity 
of graphene can contribute to the supply of electrons for oxidation around defects, instead of serving as a gas barrier $[9,10]$. In addition, if the number of layers of graphene increases to meet the high criteria of modern packaging applications, an optical loss results because graphene reduces the transmittance by $\sim 2.3 \%$ per layer [11]. This reduced transmittance adversely affects the optical properties of organic devices, such as light extraction in light emitting diodes and absorption in solar cells.

Hexagonal boron nitride (h-BN) monolayers have recently emerged as an alternative. An h-BN monolayer has several merits as a gas barrier. First, h-BN monolayers have good dielectric properties which can prevent electron transfer for oxidation and protect from strong electric shock $[10,12]$. Second, h-BN monolayers have great transparency due to the wide energy bandgap $(\sim 6 \mathrm{eV})[13,14]$. Furthermore, h-BN monolayers possess the stable material properties and high thermal stability needed for high-power device applications. We selected h-BN monolayers for study because of these advantages. However, one disadvantage is that water or oxygen molecules can penetrate through the defects $[15,16]$, so a technique to minimize defect density is required to enhance the gas barrier performance to the commercial level. Several methods to produce large-grain monolayer $\mathrm{h}-\mathrm{BN}$ have been reported, such as using an alloy catalyst [17], annealing the catalyst with hydrogen [18], and reducing the roughness of the catalyst by electrochemical polishing (ECP) [19].

In this work, we investigated the gas barrier performance of monolayer h-BN synthesized on electrochemically polished copper foils. Out of various available techniques for reducing the defect density of h-BN monolayers, ECP of copper foil was utilized because it is cheap and simple, and thus suitable for mass production. Scanning electron microscopy (SEM), Raman spectroscopy, and X-ray photoelectron spectroscopy (XPS) measurements were conducted to verify the growth of h-BN monolayers. To study the spatial distribution of the defects and the defect state, atomic force microscopy (AFM) and contact angle analysis of h-BN monolayers were performed. Finally, we measured water vapor transmission rate (WVTR) and water vapor permeability of h-BN monolayers. The performance of an h-BN monolayer as gas barrier on copper foils with ECP was comparable to that of a graphene monolayer [7].

\section{Methods}

\subsection{Electro-Chemical Polishing}

To planarize the surface of the copper foils (Alfar Aesar, 046986.RF $0.025 \mathrm{~mm}$ thick), ECP was conducted in a solution with phosphoric acid (Sigma-Aldrich, Saint Louis, MO, USA, $85 \mathrm{wt}$ \% in $\mathrm{H}_{2} \mathrm{O}, 99.99 \%$ trace metals basis, 345245-100 ML) and water at $1.8 \mathrm{~V}$ for $10 \mathrm{~min}$ using $\mathrm{Cu}$ plate as a cathode and copper foils $(100 \mathrm{~mm} \times 100 \mathrm{~mm})$ as a working electrode, as shown in Figure S1 of the supporting information.

\subsection{Synthesis of h-BN Layers}

The h-BN layers investigated in this work were synthesized on copper foils (Nippon Mining Co. Ltd., Hitachishi, Japan) via chemical vapor deposition (CVD). Borazine $\left(\mathrm{B}_{3} \mathrm{~N}_{3} \mathrm{H}_{6}\right)$ was used as a precursor for growing the h-BN and was kept in a chiller at $-10{ }^{\circ} \mathrm{C}$ in a canister with a bubbler system. Then, the copper foil with ECP treatments was placed at the center position of a quartz tube and heated by a split-tube furnace. The annealing process, under 15 standard cubic centimeters per minute $(\mathrm{sccm})$ of $\mathrm{H}_{2}$ gas at low pressure (specific value), was performed at $1040^{\circ} \mathrm{C}$ for $60 \mathrm{~min} .0 .3 \mathrm{sccm}$ of borazine and $70 \mathrm{sccm}$ of $\mathrm{H}_{2}$ were supplied at $1040{ }^{\circ} \mathrm{C}$ for $90 \mathrm{~s}$ under a growth pressure of $5 \mathrm{mTorr}$. These are the optimal conditions for obtaining high quality and uniform h-BN, as shown in Figure S2 of the supporting information. As a final step, the samples were rapidly cooled down to room temperature under a hydrogen atmosphere. The as-grown $\mathrm{h}-\mathrm{BN}$ monolayers were transferred by a method similar to graphene transfer onto various substrates, such as PET film, glass, and $\mathrm{SiO}_{2} / \mathrm{Si}$, to characterize and investigate the gas barrier properties of the h-BN. Details can be found in our previous report [7]. 


\subsection{Structure Characterizations}

Field emission scanning electron microscopy (FESEM, Quanta 200 FEG, FEI Company, Hillsboro, OR, USA) was used to observe the domain and surface morphology of the h-BN studied in this work. The quality of graphene was characterized by Raman spectroscopy (RX210 Analyser, Renishaw, Wotton-under-Edge, UK) using the $514 \mathrm{~nm}$ line of an Ar ion laser as an excitation source. X-ray photoelectron spectroscopy (XPS, K-Alpha spectrophotometer, Thermo Fisher, Waltham, MA, USA) was conducted with an AXIS Ultra DLD model with a monochromatic $\mathrm{Al} \mathrm{K} \alpha$ line at $1486.69 \mathrm{eV}$. The surface topography of h-BN on copper foils after a film-induced frustrated etching (FIFE) test was investigated with an atomic force microscope (AFM, XE-200 System, PSIA, Suwon, Korea) in tapping mode. The contact angle was measured using a water contact measurement (PHX300, Surface Electro Optics, Suwon, Korea). The water vapor transmission rate (WVTR) was evaluated by a commercial AQUATRAN model 3 WVTR analyzer (MOCON, Minneapolis, MN, USA) at room temperature under $1 \mathrm{~atm}$.

\section{Results and Discussion}

Figure 1 shows SEM images of h-BN monolayers on copper foils with and without ECP treatment. In both cases, h-BN monolayer fully covered the copper foils after $90 \mathrm{~s}^{\prime}$ growth (Figure 1a,b). In these samples, wrinkles were commonly observed for thermal stress minimization. They could stem from the nucleation of defects on the step edges of $\mathrm{Cu}$ terraces during cooling process, and their presence is indirect evidence of the successful continuous growth of h-BN monolayer [20].

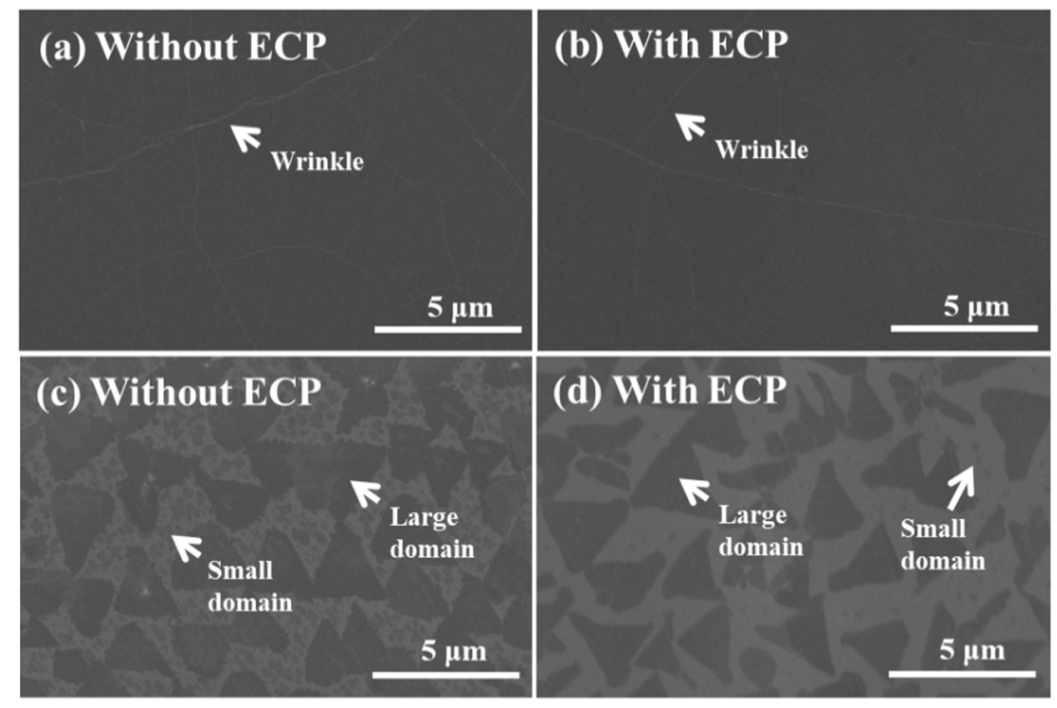

Figure 1. Scanning electron microscopic images of h-BN monolayers synthesized on copper foils $(\mathbf{a}, \mathbf{b})$ for 90 seconds and $(\mathbf{c}, \mathbf{d})$ for 60 seconds; $(\mathbf{a}, \mathbf{c})$ are without ECP treatment and $(\mathbf{b}, \mathbf{d})$ are with ECP treatment.

Once the copper foils were fully covered by h-BN monolayers, it was not possible to analyze the difference in the growth of h-BN monolayers on the two copper foil samples with and without ECP. To investigate how ECP influences the growth of an h-BN monolayer, we grew h-BN monolayers for a shorter time: $60 \mathrm{~s} \mathrm{(Figure} \mathrm{1c,d).} \mathrm{At} \mathrm{the} \mathrm{initial} \mathrm{stage} \mathrm{of}$ growth, we could detect small domains as well as large ones in both samples. The shape of the small domains was random, while that of the large domains was triangular. The areal number density of domains was noticeably different in the two cases; it was higher on copper foil without ECP than with ECP. The root-mean-square (RMS) roughness of the copper foils was measured by AFM (Figure S3). The RMS roughness of copper foils with $\mathrm{ECP}$ was measured to be $37 \mathrm{~nm}$ while that of copper foils without ECP was $78 \mathrm{~nm}$, which is consistent with SEM images. It is well known that surface irregularities such as wrinkles, 
steps, grain boundaries, and defects have much higher surface energy than a flat surface, and such features could serve as nucleation sites [21-23]. Hence, the higher irregularity of the surface of copper foils without ECP caused a higher areal nucleation density of h-BN monolayer than copper foils with ECP [7]. If the areal number density of initial domains is higher, it will lead to a higher defect density when the domains eventually merge as the growth proceeds [24-26]. As a result, h-BN monolayers grown on copper foils with ECP are expected to show better gas barrier performance owing to a lower defect density.

Figure 2a shows Raman spectra of the h-BN monolayers grown on copper foils without ECP (black line) and with ECP (red line). The peaks observed at $1369.8 \mathrm{~cm}^{-1}$ from both the samples are indicative of an h-BN monolayer [27,28]. Typically, to compare the crystallinity of h-BN monolayers, the intensities of the spectra are compared. However, it would be hard to compare the crystallinity directly using this method because of the scattering of the laser spot by the surface roughness of the copper foils. Instead, we compared the crystalline quality of h-BN monolayers on copper foils based on the full width at half maximum (FWHM). The FWHM values of h-BN monolayer grown on copper foils without ECP and with ECP were $\sim 25.02 \mathrm{~cm}^{-1}$ and $\sim 17.41 \mathrm{~cm}^{-1}$ respectively, indicating that the crystallinity of h-BN monolayers on copper foils with ECP was higher than that of h-BN monolayers on copper foils without ECP. To further confirm the growth of the h-BN monolayers, we measured the 1s core level XPS spectra of boron and nitrogen in the h-BN monolayers on copper foils with and without ECP (Figure $2 b, c)$. From both samples, peaks in the XPS spectra were observed at $190.8 \mathrm{eV}$, which corresponds to the binding energy of boron atoms $[29,30]$. Peaks were also observed at $\sim 398.2 \mathrm{eV}$ in both samples. This energy corresponds to the binding energy of nitride atoms $[29,30]$.

(a)

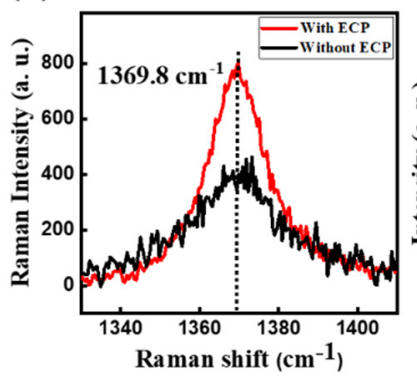

(b)

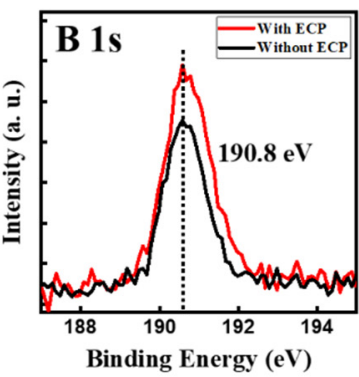

(c)

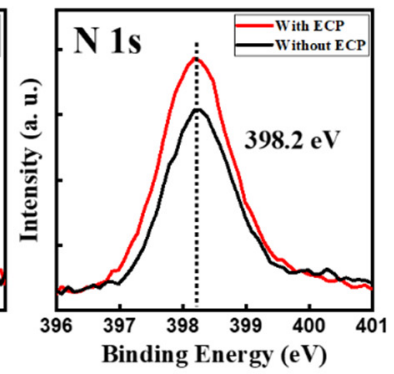

Figure 2. (a) Raman spectra of h-BN monolayers on copper foils without ECP (black line) and with ECP (red line). $(\mathbf{b}, \mathbf{c})$ show the 1s core level XPS spectra of boron and nitrogen of h-BN monolayers on copper foils without ECP and with ECP, respectively.

To investigate the spatial distribution of the defects, the surface morphology of h-BN monolayers on copper foils without ECP and with ECP was examined by AFM (Figure 3a,b). Before the measurements, a film-induced frustrated etching (FIFE) was completed to reveal the spatial distribution of the defects. The etched fit densities for h-BN monolayers on copper foils without ECP and with ECP were estimated to be $1.3 \times 108 / \mathrm{cm}^{2}$ and $3 \times 107 / \mathrm{cm}^{2}$, respectively (Figure 3c). In addition, etched to total area ratios were obtained by an image processing program (Image J 1.52p, National Institute of Health, Bethesda, MD, USA) based on a randomly selected area (indicated by a white rectangle in Figure $3 a, b$ ) of the AFM images of both samples [31]. The etched to total area ratios of h-BN monolayers on copper foils without ECP and with ECP were $23 \%$ and $3 \%$, respectively. It is obvious that the h-BN monolayer on copper foils with ECP had a lower etched pit density with a smaller etched area, indicating that the ECP methods significantly reduced the defect density. 

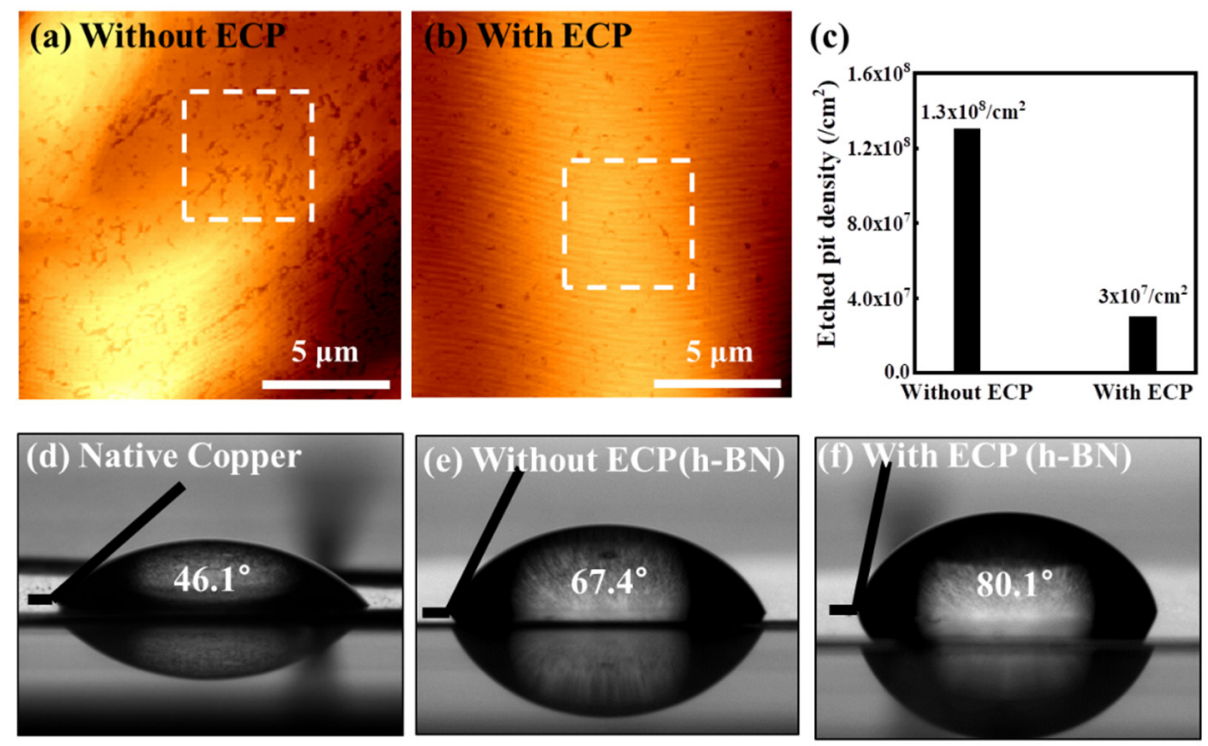

Figure 3. AFM images of the surface of h-BN monolayers on copper foils (a) without ECP and (b) with ECP after a film-induced frustrated etching. (c) Etched pit density observed from AFM images. (d-f) Contact angles of water on a (d) native copper foil, (e) an h-BN monolayer on copper foils without ECP, and (f) that with ECP, respectively.

We also evaluated contact angles of water on native copper foils, an h-BN monolayer on copper foil without ECP, and one with ECP, respectively (Figure $3 \mathrm{~d}-\mathrm{f}$ ). Contact angle analysis is a fast and straightforward technique for estimating the defect density of $2 \mathrm{D}$ materials such as graphene and h-BN monolayers [32-34]. The measured contact angle of water on a native copper foil was $46.1^{\circ}$, which was low due to the high surface energy between water and copper foil. The contact angles were higher in the other samples as the copper substrates were passivated by the h-BN monolayers; they were found to be $67.4^{\circ}$ without ECP and $80.1^{\circ}$ with ECP. It is well known that $\mathrm{h}-\mathrm{BN}$ is totally charge neutral and nonpolar, satisfying the octet rule. However, when the defects are created, the resulting dangling bonds break the charge neutrality. Because of this, defects in h-BN monolayer contribute to a change in the contact angle by increasing the surface energy and surface polarities [35]. The h-BN monolayer on the copper foil without ECP had higher surface energy than that with ECP, implying that the defect density of h-BN monolayer was higher on the copper foil without ECP than that with ECP. This result is consistent with our discussion of defect density based on the results of SEM, Raman spectroscopy, and AFM analyses.

WVTR was measured with varying time for bare polyethylene terephthalate (PET), an h-BN monolayer without ECP on PET, an h-BN monolayer with ECP on PET, and graphene on PET by a commercial MOCON's proprietary AQUATRAN model 3 WVTR analyzer (Figure 4a). Recently, we studied the gas barrier properties of graphene grown on copper foils with ECP treatment [7]. Here, we compare the gas barrier performance of h-BN monolayers and graphene. All four samples arrived at a steady state of WVTR within 1 day. The WVTR values of $h-B N$ monolayers without and with ECP were measured to be, respectively, 0.798 and $0.774 \mathrm{~g} \mathrm{~m}^{-2}$ day $^{-1}$. These values were slightly higher than that of graphene on PET $\left(0.728 \mathrm{~g} \mathrm{~m}^{-2}\right.$ day $\left.^{-1}\right)$, but significantly lower than that of bare PET $\left(1.101 \mathrm{~g} \mathrm{~m}^{-2} \mathrm{day}^{-1}\right)$. The gas barrier properties of $\mathrm{h}-\mathrm{BN}$ are slightly lower than that of graphene, but after a long term, we believe that gas barrier properties of h-BN will be improved than that of graphene. This is because $\mathrm{h}-\mathrm{BN}$ is not easily oxidized by the absence of itinerant electron. Between h-BN monolayers, the one with ECP showed a lower WVTR value than that without ECP. We expect that the lower WVTR value of the h-BN monolayer with ECP is associated with its reduced defects and grain boundaries. The results indicate that the h-BN monolayer with ECP successfully acted as a protective layer for PET against 
water. Recently, the gas-barrier performance of a wafer-scale single-crystal h-BN monolayer on PET was reported, and was $0.60 \mathrm{~g} \mathrm{~m}^{-2}$ day $^{-1}$ [36]. A single-crystal h-BN monolayer is ideal for the gas barrier application since it can minimize the diffusion of gas molecules through the grain boundary. However, the synthesis of a single-crystalline h-BN monolayer is very expensive and not generic yet. Thus, our approach to use poly-crystalline h-BN monolayers composed of large grains as gas barriers is more realistic since they are more economical and can be readily mass produced.
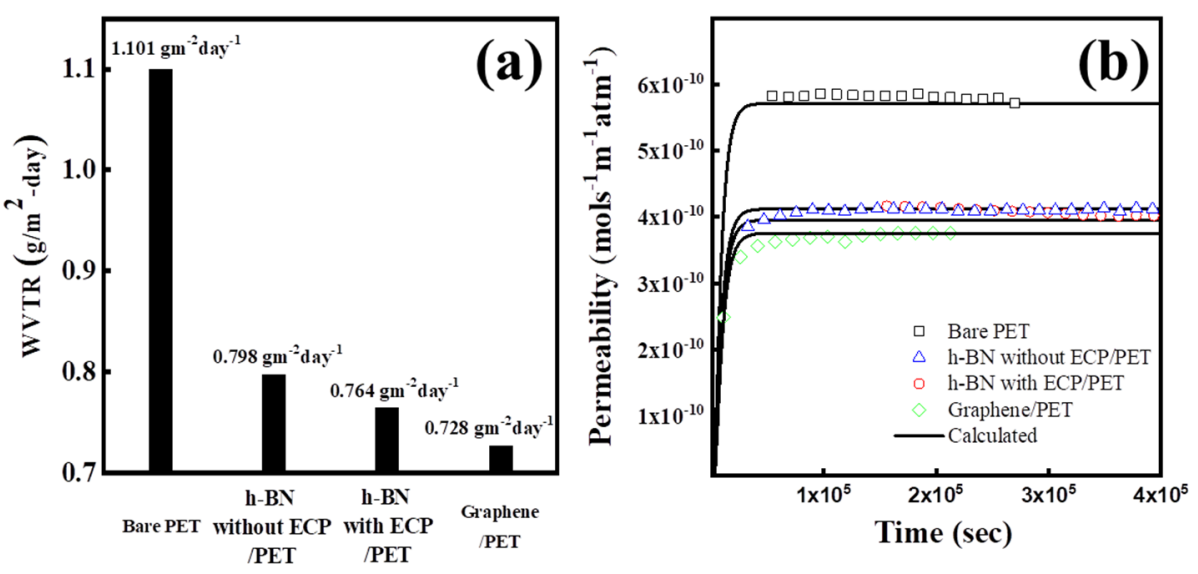

Figure 4. (a) WVTR and (b) water vapor permeability of pure PET, an h-BN monolayer without ECP on PET, an h-BN monolayer with ECP on PET, graphene on PET.

The water permeability of samples can be obtained from Equation (1) [37]

$$
P^{\circ}=P\left(\frac{4 d^{2}}{\pi D t}\right)^{0.5} \sum_{n=0}^{\infty} \exp \left[\frac{-d^{2}}{4 D t}(2 n+1)^{2}\right]
$$

Here, $\mathrm{J}$ and $\mathrm{J}_{\mathrm{s}}$ are the water vapor molar flux at time $\mathrm{t}$ and at steady state, respectively, $\mathrm{d}$ is the sample thickness, $\mathrm{P}$ is the permeability, $\mathrm{D}$ is the diffusivity, $\mathrm{S}$ is the solubility, and $\mathrm{P}^{0}=\mathrm{Jd}, \mathrm{P}=\mathrm{J}_{\mathrm{S}} \mathrm{d}=\mathrm{SD}$. The permeability, diffusivity, and solubility of all samples were determined from the best fitting of Fick's second law of diffusion in Figure 4b. Table 1 shows the estimated permeability, diffusivity and solubility of bare PET, a h-BN monolayer without ECP on PET, a h-BN monolayer with ECP on PET, and graphene on PET, respectively. The permeabilities were $7.430 \times 10^{-14}, 5.36 \times 10^{-14}, 5.14 \times 10^{-14}$, and $4.88 \times 10^{-14} \mathrm{mols}^{-1} \mathrm{~m}^{-1} \mathrm{~atm}^{-1}$, respectively. Interestingly, the decreased permeability was not correlated to the diffusivity; the diffusivity of all samples was nearly identical. Meanwhile, the solubilities of the h-BN monolayer without ECP on PET, the h-BN monolayer with ECP on PET, and graphene on PET were decreased by $25.4 \%, 26.4 \%$, and $27.0 \%$, respectively, compared to the solubility of bare PET. These results indicate that when adopting 2D material as a gas barrier, the reduction of permeability is caused by reduced solubility rather than the diffusion path blocking effect.

Table 1. Estimated permeability, diffusivity and solubility of bare PET, h-BN monolayer without ECP on PET, h-BN monolayer with ECP on PET, and graphene on PET.

\begin{tabular}{ccccc}
\hline & Bare PET & h-BN Without ECP/PET & h-BN with ECP/PET & Graphene/PET \\
\hline $\begin{array}{c}\text { Permeability } \\
{\left[\times 10^{-14} \mathrm{~mol} \mathrm{~s}^{-1} \mathrm{~m}^{-1} \mathrm{~atm}^{-1}\right]}\end{array}$ & 7.43 & 5.36 & 5.14 & 4.88 \\
\hline $\begin{array}{c}\text { Diffusivity } \\
{\left[\times 10^{-14} \mathrm{~m}^{2} \mathrm{~s}^{-1}\right]}\end{array}$ & 0.30 & 0.28 & 0.28 & 0.27 \\
\hline $\begin{array}{c}\text { Solubility } \\
{\left[\mathrm{mol} \mathrm{m}^{-3} \mathrm{~atm}^{-1}\right]}\end{array}$ & 24.8 & 18.5 & 18.2 & 18.1 \\
\hline
\end{tabular}




\section{Conclusions}

In summary, we investigated the gas barrier performance of h-BN monolayers on copper foils with ECP treatment. The ECP treatment of copper foils significantly reduced the defect density of h-BN monolayers. WVTR and permeability of h-BN monolayers on PET were significantly lower than those of bare PET and were comparable to those of graphene on PET. In addition, the ECP treatment of copper foils resulted in improved gas barrier performance of h-BN monolayers. The results demonstrate the potential of h-BN monolayers as gas barriers in organic-based optoelectronic devices.

Supplementary Materials: The following are available online at https://www.mdpi.com/article/ 10.3390/app11104599/s1, Figure S1: Schematics and experimental conditions of electrochemical polishing for $\mathrm{Cu}$ foils. Figure S2: The optimization process of synthesis conditions for high quality and uniform h-BN. Figure S3: AFM images and RMS roughness of $\mathrm{Cu}$ foils (a) without ECP and (b) with ECP, respectively.

Author Contributions: Conceptualization, C.H.L., G.B.C., and J.L. (Jaegeun Lee); Investigation, C.H.L. and J.L. (Jongho Lee); Data curation, C.H.L. and T.H.S.; Writing-original draft, C.H.L.; Writing, G.B.C.; Methodology, G.B.C., Validation, E.M.K., H.G.M., and Y.A.K.; Formal analysis, E.M.K., J.L. (Jongho Lee), M.J.K., and Y.A.K.; Writing-review \& editing, J.L. (Jaegeun Lee) and T.H.S.; Resources, H.G.M. and M.J.K.; Supervision, T.H.S.; Project administration, T.H.S. All authors have read and agreed to the published version of the manuscript.

Funding: This research was supported by the Korea Institute of Industrial Technology (KITECH) and by the National Research Foundation of Korea (NRF) funded by the Ministry of Education, Science and Technology (2019RM3F5A1A02092650). And Y.A.K acknowledges support from the Nano-Material Technology Development Program through the National Research Foundation of Korea (NRF), funded by Ministry of Science and ICT (2017M3A7B4014045).

Institutional Review Board Statement: Not applicable.

Informed Consent Statement: Not applicable.

Data Availability Statement: Not applicable.

Conflicts of Interest: The authors declare no conflict of interest.

\section{References}

1. Wang, X.; Zhou, D.; Huang, J.; Yu, J. High performance organic ultraviolet photodetector with efficient electroluminescence realized by a thermally activated delayed fluorescence emitter. Appl. Phys. Lett. 2015, 107, 043303. [CrossRef]

2. Pradana, A.; Gerken, M. Photonic crystal slabs in flexible organic light-emitting diodes. Photon. Res. 2015, 3, 32-37. [CrossRef]

3. Yu, D.; Yang, Y.-Q.; Chen, Z.; Tao, Y.; Liu, Y.-F. Recent progress on thin-film encapsulation technologies for organic electronic devices. Opt. Commun. 2016, 362, 43-49. [CrossRef]

4. Xiao, W.; Hui, D.Y.; Zheng, C.; Yu, D.; Qiang, Y.Y.; Ping, C.; Xiang, C.L.; Yi, Z. A flexible transparent gas barrier film employing the method of mixing ALD/MLD-grown Al2O3 and alucone layers. Nanoscale Res. Lett. 2015, 10, 130. [CrossRef] [PubMed]

5. Xie, X.; Rieth, L.; Williams, L.; Negi, S.; Bhandari, R.; Caldwell, R.; Sharma, R.; Tathireddy, P.; Solzbacher, F. Long-term relia-bility of $\mathrm{Al} 2 \mathrm{O} 3$ and Parylene $\mathrm{C}$ bilayer encapsulated Utah electrode array based neural interfaces for chronic implantation. J. Neural Eng. 2014, 11, 26016. [CrossRef] [PubMed]

6. Ding, D.; Hibino, H.; Ago, H. Grain Boundaries and Gas Barrier Property of Graphene Revealed by Dark-Field Optical Microscopy. J. Phys. Chem. C 2018, 122, 902-910. [CrossRef]

7. Seo, T.H.; Lee, S.; Cho, H.; Chandramohan, S.; Suh, E.-K.; Lee, H.S.; Bae, S.K.; Kim, S.M.; Park, M.; Lee, J.K.; et al. Tailored CVD graphene coating as a transparent and flexible gas barrier. Sci. Rep. 2016, 6, 24143. [CrossRef]

8. Cui, C.; Lim, A.T.O.; Huang, J. A cautionary note on graphene anti-corrosion coatings. Nat. Nanotechnol. 2017, 12, 834-835. [CrossRef]

9. Schriver, M.; Regan, W.; Gannett, W.J.; Zaniewski, A.M.; Crommie, M.F.; Zettl, A. Graphene as a Long-Term Metal Oxidation Barrier: Worse Than Nothing. ACS Nano 2013, 7, 5763-5768. [CrossRef]

10. Tanjil, R.-E.; Jeong, Y.; Yin, Z.; Panaccione, W.; Wang, M.C. Ångstrom-Scale, Atomically Thin 2D Materials for Corrosion Mitigation and Passivation. Coatings 2019, 9, 133. [CrossRef]

11. Luo, S.; Wang, Y.; Tong, X.; Wang, Z. Graphene-based optical modulators. Nanoscale Res. Lett. 2015, 10, 199. [CrossRef] [PubMed]

12. Laturia, A.; Van De Put, M.L.; Vandenberghe, W.G. Dielectric properties of hexagonal boron nitride and transition metal dichalcogenides: From monolayer to bulk. 2D Mater. Appl. 2018, 2, 6. [CrossRef] 
13. Wang, J.; Ma, F.; Sun, M. Graphene, hexagonal boron nitride, and their heterostructures: Properties and applications. RSC Adv. 2017, 7, 16801-16822. [CrossRef]

14. Sun, J.; Lu, C.; Song, Y.; Ji, Q.; Song, X.; Li, Q.; Zhang, Y.; Zhang, L.; Kong, J.; Liu, Z. Recent progress in the tailored growth of two-dimensional hexagonal boron nitride via chemical vapour deposition. Chem. Soc. Rev. 2018, 47, 4242-4257. [CrossRef] [PubMed]

15. Boutilier, M.S.H.; Sun, C.; O’Hern, S.C.; Au, H.; Hadjiconstantinou, N.G.; Karnik, R. Implications of Permeation through Intrinsic Defects in Graphene on the Design of Defect-Tolerant Membranes for Gas Separation. ACS Nano 2014, 8, 841-849. [CrossRef]

16. Giesbers, A.; Bouten, P.; Cillessen, J.; Van Der Tempel, L.; Klootwijk, J.; Pesquera, A.; Centeno, A.; Zurutuza, A.; Balkenende, A. Defects, a challenge for graphene in flexible electronics. Solid State Commun. 2016, 229, 49-52. [CrossRef]

17. Lu, G.; Wu, T.; Yuan, Q.; Wang, H.; Wang, H.; Ding, F.; Xie, X.; Jiang, M. Synthesis of large single-crystal hexagonal boron nitride grains on Cu-Ni alloy. Nat. Commun. 2015, 6, 6160. [CrossRef] [PubMed]

18. Cho, H.; Park, S.; Won, D.I.; Kang, S.O.; Pyo, S.S.; Kim, D.I.; Kim, S.M.; Kim, H.C.; Kim, M.J. Growth kinetics of white gra-phene (h-BN) on a planarised Ni foil surface. Sci. Rep. 2015, 5, 11985. [CrossRef]

19. Tay, R.Y.; Griep, M.H.; Mallick, G.; Tsang, S.H.; Singh, R.S.; Tumlin, T.; Teo, E.H.T.; Karna, S.P. Growth of large single-crystalline two-dimensional boron nitride hexagons on electropolished copper. Nano Lett. 2014, 14, 839-846. [CrossRef]

20. Li, X.; Cai, W.; An, J.; Kim, S.; Nah, J.; Yang, D.; Piner, R.; Velamakanni, A.; Jung, I.; Tutuc, E.; et al. Large-Area Synthesis of High-Quality and Uniform Graphene Films on Copper Foils. Science 2009, 324, 1312-1314. [CrossRef]

21. Braeuninger-Weimer, P.; Brennan, B.; Pollard, A.J.; Hofmann, S. Understanding and Controlling Cu-Catalyzed Graphene Nucleation: The Role of Impurities, Roughness, and Oxygen Scavenging. Chem. Mater. 2016, 28, 8905-8915. [CrossRef] [PubMed]

22. Han, G.H.; Güneş, F.; Bae, J.J.; Kim, E.S.; Chae, S.J.; Shin, H.-J.; Choi, J.-Y.; Pribat, D.; Lee, Y.H. Influence of Copper Morphology in Forming Nucleation Seeds for Graphene Growth. Nano Lett. 2011, 11, 4144-4148. [CrossRef] [PubMed]

23. Griep, M.H.; Sandoz-Rosado, E.; Tumlin, T.M.; Wetzel, E. Enhanced Graphene Mechanical Properties through Ultrasmooth Copper Growth Substrates. Nano Lett. 2016, 16, 1657-1662. [CrossRef] [PubMed]

24. Tian, W.; Li, W.; Yu, W.; Liu, X. A Review on Lattice Defects in Graphene: Types, Generation, Effects and Regulation. Micromachines 2017, 8, 163. [CrossRef]

25. Sun, X.-Y.; Hu, H.; Cao, C.; Xu, Y.-J. Anisotropic vacancy-defect-induced fracture strength loss of graphene. RSC Adv. 2015, 5, 13623-13627. [CrossRef]

26. Yang, B.; Wang, S.; Guo, Y.; Yuan, J.; Si, Y.; Zhang, S.; Chen, H. Strength and failure behavior of a graphene sheet containing bi-grain-boundaries. RSC Adv. 2014, 4, 54677-54683. [CrossRef]

27. Stehle, Y.; Meyer, I.H.M.; Unocic, R.R.; Kidder, M.; Polizos, G.; Datskos, P.G.; Jackson, R.; Smirnov, S.N.; Vlassiouk, I.V. Synthesis of Hexagonal Boron Nitride Monolayer: Control of Nucleation and Crystal Morphology. Chem. Mater. 2015, 27, 8041-8047. [CrossRef]

28. Mahvash, F.; Eissa, S.; Bordjiba, T.; Tavares, A.C.; Szkopek, T.; Siaj, M. Corrosion resistance of monolayer hexagonal boron nitride on copper. Sci. Rep. 2017, 7, 42139. [CrossRef]

29. Lee, S.H.; Jeong, H.; Okello, O.F.N.; Xiao, S.; Moon, S.; Kim, D.Y.; Kim, G.Y.; Lo, J.I.; Peng, Y.C.; Cheng, B.M.; et al. Improvements in structural and optical properties of wafer-scale hexagonal boron nitride film by post-growth annealing. Sci. Rep. 2019, 9, 10590. [CrossRef]

30. Haider, A.; Ozgit-Akgun, C.; Goldenberg, E.; Okyay, A.K.; Biyikli, N. Low-Temperature Deposition of Hexagonal Boron Nitride via Sequential Injection of Triethylboron and N2/H2 Plasma. J. Am. Ceram. Soc. 2014, 97, 4052-4059. [CrossRef]

31. Abràmoff, M.D.; Magalhães, P.J.; Ram, S.J. Image Processing with ImageJ. Biophotonics Int. 2004, 11, 36-42. [CrossRef]

32. Belyaeva, L.A.; van Deursen, P.M.G.; Barbetsea, K.I.; Schneider, G.F. Hydrophilicity of Graphene in Water through Trans-parency to Polar and Dispersive Interactions. Adv. Mater. 2018, 30, 1703274. [CrossRef]

33. Prydatko, A.V.; Belyaeva, L.A.; Jiang, L.; Lima, L.M.C.; Schneider, G.F. Contact angle measurement of free-standing squaremillimeter single-layer graphene. Nat. Commun. 2018, 9, 4185. [CrossRef] [PubMed]

34. Parobek, D.; Liu, H. Wettability of graphene. 2D Mater. 2015, 2. [CrossRef]

35. Annamalai, M.; Gopinadhan, K.; Han, S.A.; Saha, S.; Park, H.J.; Cho, E.B.; Kumar, B.; Patra, A.; Kim, S.-W.; Venkatesan, T. Surface energy and wettability of van der Waals structures. Nanoscale 2016, 8, 5764-5770. [CrossRef] [PubMed]

36. Lee, J.S.; Choi, S.H.; Yun, S.J.; Kim, Y.I.; Boandoh, S.; Park, J.-H.; Shin, B.G.; Ko, H.; Lee, S.H.; Lee, Y.H.; et al. Wafer-scale single-crystal hexagonal boron nitride film via self-collimated grain formation. Science 2018, 362, 817-821. [CrossRef]

37. Kim, H.M.; Lee, J.K.; Lee, H.S. Transparent and high gas barrier films based on poly(vinyl alcohol)/graphene oxide composites. Thin Solid Films 2011, 519, 7766-7771. [CrossRef] 